\title{
Altered pulmonary capillary permeability in immunosuppressed guinea pigs infected with Legionella pneumophila serogroup 1
}

\author{
$\mathrm{XU} \mathrm{CAI}^{1}, \mathrm{NA} \mathrm{YU}^{1}$, JIANGWEI MA $^{1}$, WEN-YANG LI ${ }^{1}$, MINGTAO XU $^{1}$, ERRAN LI ${ }^{1}$, \\ MIN ZHANG $^{2}$, WEI WANG ${ }^{1}$, YU CHEN ${ }^{3}$ and JIAN KANG ${ }^{1}$ \\ ${ }^{1}$ Department of Respiratory Medicine, The First Hospital of China Medical University, Shenyang, Liaoning 110001; \\ ${ }^{2}$ Department of Respiratory Medicine, Heilongjiang Provincial Hospital, Harbin, Heilongjiang 150036; \\ ${ }^{3}$ Department of Respiratory Medicine, Shengjing Hospital of China Medical University, \\ Shenyang, Liaoning 110004, P.R. China
}

Received November 25, 2018; Accepted August 16, 2019

DOI: $10.3892 /$ etm.2019.8102

\begin{abstract}
In immunosuppressed hosts, Legionella pneumophila $(\mathrm{Lp})$ infection usually develops into severe pneumonia, which is pathologically characterized by increased vascular permeability and pulmonary edema. At present, mechanisms associated with changes in pulmonary capillary permeability (PCP) and the pathogenesis of pulmonary edema in immunosuppressed hosts with $L p$ infection are unclear. Therefore, in the present study an animal model of normal and immunosuppressed guinea pigs infected with Lp was established. An isolated perfused lung system was used to investigate the extent of changes in PCP. Pathological and immunofluorescence examinations were performed to explore the mechanism underlying these changes. The results indicated that PCP increased with the highest magnitude in immunosuppressed guinea pigs infected with Lp, with repeated ANOVA indicating synergism between infection and immunosuppression $(\mathrm{P}=0.0444)$. Hematoxylin and eosin staining and electron microscopy revealed more severe morphological damages in the lung tissues and pulmonary capillaries of the immunosuppressed animals infected
\end{abstract}

Correspondence to: Professor Jian Kang, Department of Respiratory Medicine, The First Hospital of China Medical University, 155 Nanjing North Street, Shenyang Liaoning 110001, P.R. China

E-mail: kangjian58@163.com

Professor Yu Chen, Department of Respiratory Medicine, Shengjing Hospital of China Medical University, 36 Sanhao Street, Shenyang, Liaoning 110004, P.R. China

E-mail: chenyusy@hotmail.com

Abbreviations: Lp, Legionella pneumophila; PCP, pulmonary capillary permeability; PAP, pulmonary artery pressure

Key words: immunosuppression, Legionella pneumophila serogroup 1, pulmonary capillary permeability, pulmonary artery pressure, isolated lung perfusion with Lp compared with normal animals infected with Lp. Immunofluorescence analysis showed that immunosuppression reduced the expression of the vascular endothelial cell junction protein VE-cadherin $(\mathrm{P}=0.027)$. Following Lp infection, VE-cadherin expression was significantly lower in the immunosuppressed guinea pigs compared with their immunocompetent counterparts $(\mathrm{P}=0.001)$. These results suggest that immunosuppression combined with Lp infection induces more significant damage to pulmonary capillaries compared with Lp infection alone, resulting in a significantly increased PCP.

\section{Introduction}

Legionella pneumophila (Lp), a gram-negative intracellular bacterium, is a common pathogen that causes community-acquired and hospital-acquired pneumonia. It is one of the three most common causative agents of severe pneumonia with an incidence of $2-9 \%$ and a mortality rate of $10 \%$ in Europe and North America (1). In particular, Immunosuppression as a result of a number of factors, including chronic disease, malignant tumors or the administration of immunosuppressive drugs is an important risk factor for Lp infection $(2,3)$. Immunosuppressed hosts infected with Lp have an elevated risk of developing severe pneumonia, with the mortality rate of the disease potentially reaching $20-70 \%$ in these cases $(2,4)$.

The most common pathophysiological characteristics associated with severe pneumonia are increased pulmonary capillary permeability (PCP) and pulmonary edema (5). It is well documented that infectious pulmonary edema is primarily caused by increased PCP, which is also an important indicator of the severity of lung injury (5). Lp infection induces severe pneumonia more readily in immunosuppressed hosts compared with immunocompetent hosts (6). However, whether the increase of PCP or pulmonary edema are more severe in Lp-infected immunosuppressed hosts compared with hosts with normal immune function remains unclear.

Damage to the vascular endothelial barrier leads to the exudation of protein-rich pulmonary edema fluid (7). 
Vascular endothelial cadherin (VE-cadherin) is an important component for maintaining PCP balance. VE-cadherin internalization increases vascular permeability and endothelial cell migration, leading to pulmonary edema (8).

Currently, most studies of Lp infection in immunocompromised hosts have been case reports $(9,10)$, and there are insufficient data on the differences in Lp infection between immunocompromised hosts and those with normal immune function. It is conceivable to speculate that injury caused by Lp infection is more severe in immunosuppressed hosts compared with hosts with normal immune function, and that the major pathological characteristic is the increase in PCP. To test this hypothesis, in the present study an IL-2 isolated perfused system was applied to investigate the changes in the ex vivo weights of lung tissues from Lp-infected immunosuppressed guinea pigs and those with normal immunity. Variations in PCP in Lp-infected animals with different immune status were also evaluated.

\section{Materials and methods}

Laboratory animals, immunosuppression induction and grouping. A total of 144 specific-pathogen-free male Hartley guinea pigs (age, 4-5 weeks; weight range, 300-350 g) were purchased from Beijing Vital River Laboratory Animal Technology Co., Ltd. Each guinea pig was raised in an individually ventilated cag and maintained at $23^{\circ} \mathrm{C}$ environment under a $12 \mathrm{~h}$ light/dark cycle. The animals had free access to sterile feed and water. This experiment was approved by the Experimental Animal Welfare and Ethics Committee of the Chinese Medical University (Shenyang, China).

The experimental animals were divided into four groups: Control, Lp-infected, immunosuppressed and immunosuppressed + Lp-infected (Fig. 1). Immunosuppressed guinea pigs (11) were obtained by treating the guinea pigs with Triamcinolone acetonide (Beijing Solarbio Science \& Technology Co., Ltd) and cyclophosphamide (Cayman Chemical Company). A subcutaneous injection of triamcinolone acetonide $(20 \mathrm{mg} / \mathrm{kg})$ was administered to each animal daily for four days. On the fourth day, an intraperitoneal injection of cyclophosphamide $(300 \mathrm{mg} / \mathrm{kg})$ was also given to the same animal. This transient immunosuppressive treatment resulted in a reduction of total white blood cell counts to $<1 \times 10^{9} / 1$, with this immunosuppression lasting seven days, consistent with previous literature reports (11). On the fifth day, the animals in the Lp-infected and immunosuppressed Lp-infected groups were anesthetized with an intraperitoneal injection of sodium pentobarbital $(40 \mathrm{mg} / \mathrm{kg})$. Their neck skin was cut and $0.3 \mathrm{ml}$ Lp serogroup 1 ( $1 \times 10^{6}$ bacteria/guinea pig) suspended in saline of was injected into the main airway using a $1 \mathrm{ml}$ syringe (12). For the animals in the control and the immunosuppressed groups, $0.3 \mathrm{ml}$ sterile saline was injected into the main airway under the same conditions. All animals regained consciousness $\sim 1 \mathrm{~h}$ following Lp challenge.

Each experimental group contained 36 guinea pigs. The experiments were conducted at three time points $(24,48$ and $72 \mathrm{~h}$ after Lp infection). At each time point, 12 animals in each group were randomly selected. Of the 12 guinea pigs, six were designated for isolated lung perfusion whilst the other six were designated for histology and biochemical analyses.
In isolated lung perfusion, following anesthesia by an intraperitoneal injection of sodium pentobarbital $(40 \mathrm{mg} / \mathrm{kg})$, hearts and lungs were removed from each animal for maintenance under extracorporeal pulmonary circulation.

For histological and biochemical analyses, following anesthesia with an intraperitoneal injection of sodium pentobarbital $(40 \mathrm{mg} / \mathrm{kg})$, an incision was made in the chest of the animals along the midline. A total of $2 \mathrm{ml}$ blood was subsequently collected from the right ventricle before the lungs were extracted. Of the blood sample, $1 \mathrm{ml}$ was divided into an anticoagulant tube for the measurement of white blood cell count using Auto Hematology Analyzer (BC-2800Vet; Shenzhen Mindray Bio-Medical Electronics Co., Ltd). A section of the left lower lung tissue $\left(1 \mathrm{~mm}^{3}\right)$ was cut and fixed using $2.5 \%$ glutaraldehyde at $4^{\circ} \mathrm{C}$ for 3 days for electron microscopic analyses, whereas the remaining left lung tissues were fixed with $4 \%$ formalin (room temperature) for 2 days at room temperature for hematoxylin and eosin (H\&E) and immunofluorescence staining. Remaining blood and lung tissue samples were stored in $-80^{\circ} \mathrm{C}$.

Since the lungs were removed from the thoracic cavities of all experimental animals during the operation procedure, all animals were sacrificed.

Bacterial strain and culture. The Lp strain used in this study was clinically isolated in The Respiratory Department of Shengjing Hospital affiliated to the Chinese Medical University (Shengyang, China). The strain was verified to be of Lp serogroup 1 using slide agglutination serological test, with the protocol described as follows (Fig. S1): A total of $25 \mu \mathrm{l} \mathrm{Lp} 1$ serum was dropped onto a microscopic slide, following which a colony of Lp 1 was added into it. The slides were subsequently shaken for $1 \mathrm{~min}$ and then assessed for agglutination. Sterile water was used as control. The bacteria were cultured in Legionella CYE-Agar (Base) medium (CM0665B; Oxoid, Ltd.; Thermo Fisher Scientific, Inc.) containing Legionella BCYE growth supplement (SR0110C; Oxoid, Ltd.; Thermo Fisher Scientific, Inc.) at $37^{\circ} \mathrm{C}$ under $5 \% \mathrm{CO}_{2}$. After four days in culture, the bacteria were resuspended in sterile normal saline at a concentration of $3.33 \times 10^{6} \mathrm{cfu} / \mathrm{ml}$.

In vivo pulmonary edema. At 24, 48 and $72 \mathrm{~h}$ following infection with Lp, six animals were selected in each group with the weights of their lungs were measured to evaluate the severity of pulmonary edema in vivo.

Ex vivo lung perfusion. In the present study, the IPL-2 Isolated Perfused Lung System (Harvard Apparatus) (Fig. S2) $(13,14)$ was used to perform ex vivo lung perfusion experiment. Changes in the weights of the lungs in vivo were measured in constant-pressure perfusion mode, which was used to directly measure changes in PCP. A total of six animals in each group were selected and anesthetized with an intraperitoneal injection of $40 \mathrm{mg} / \mathrm{kg}$ pentobarbital sodium 24, 48 and $72 \mathrm{~h}$ after Lp infection (15). The trachea of each guinea pig was cut, followed by intubation and maintenance of positive pressure ventilation $\left(2-10 \mathrm{~cm} \mathrm{H}_{2} \mathrm{O}\right)$ at a respiratory rate of 60 beats $/ \mathrm{min}$. An incision was made in the abdomen from the bottom to top along the midline and the bilateral femoral arteries were 


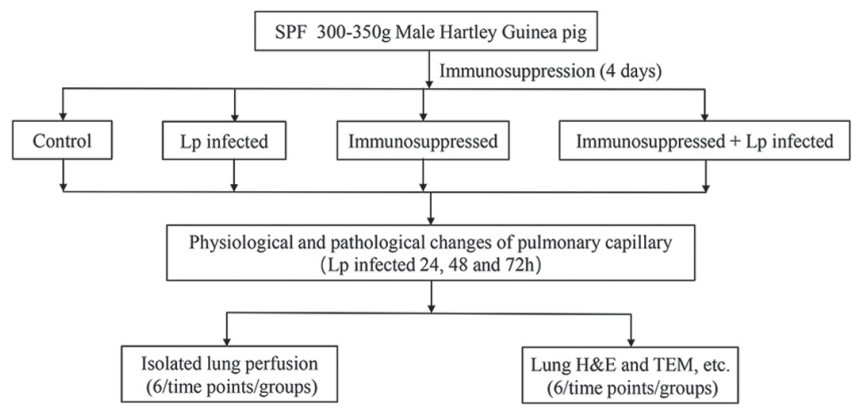

Figure 1. Flow chart of experiment. Immunosuppression: Triamcinolone acetonide $20 \mathrm{mg} / \mathrm{kg} 1-4$ days, cyclophosphamide $300 \mathrm{mg} / \mathrm{kg}$ day 4 .

cut off for exsanguination. An additional incision was made in the thoracic cavity along the midline to expose the heart and lung, and $1 \mathrm{ml} 0.9 \%$ sodium chloride solution containing 250 IU heparin sodium was injected into the right ventricle to prevent thrombosis within the pulmonary vessels. An arterial cannula was inserted into the pulmonary artery via an incision in the right ventricle whereas a venous cannula was inserted into the left atrium via an incision in the left ventricle. The lungs were subsequently separated from the surrounding tissues and incorporated into the artificial chest of IPL-2 system. The ventilation mode was changed from positive-pressure ventilation to negative-pressure (between -2 and $-10 \mathrm{cmH}_{2} \mathrm{O}$ ). Perfusion of airflow was then commenced at a rate of $5 \mathrm{ml} / \mathrm{min}$ before being gradually increased and the mode was changed to constant-pressure perfusion at $10 \mathrm{cmH}_{2} \mathrm{O}$, which is considered to be close to that of the normal average pulmonary artery pressure (PAP) (16). The ex vivo weight of the lung was then adjusted to $0 \mathrm{mg}$ and the subsequent values for the ex vivo weight of the lung was recorded every $5 \mathrm{~min}$ for $30 \mathrm{~min}$. The perfusate composition (Krebs-Henseleit solution) (15) was: $\mathrm{NaCl}, 118 \mathrm{mM}$; KCL, $4.7 \mathrm{mM} ; \mathrm{KH}_{2} \mathrm{PO}_{4}, 1.2 \mathrm{mM}$; $\mathrm{MgSO}_{4}, 1.2 \mathrm{mM} ; \mathrm{CaCl}_{2}, 2.5 \mathrm{mM}$; $\mathrm{NaHCO}_{3}, 24.9 \mathrm{mM}$; bovine serum albumin (BSA) (Amresco, LLC), 2\%; glucose, $5.56 \mathrm{mM}$; and HEPES, $12.6 \mathrm{mM}$. The temperature of the perfusate and the artificial chest was maintained at $37^{\circ} \mathrm{C}$.

Histology and immunofluorescence. Following fixation, paraffin-embedded lung tissue samples were cut into $4 \mu \mathrm{m}$ sections and stained with H\&E for a total of $6 \mathrm{~min}$ at room temperature. Inflammation area in $\mathrm{H} \& \mathrm{E}$ lung sections from six guinea pigs were analyzed and quantified by three senior pathologists, using the Image J software (version 1.48; National Institutes of Health) (17).

For immunofluorescence staining. All sections were blocked for $30 \mathrm{~min}$ using $5 \% \mathrm{BSA}$ at $20^{\circ} \mathrm{C}$. A primary anti-VE-cadherin antibody (cat. no. ab33168; 1:400; Abcam) was diluted in QuickBlock ${ }^{\mathrm{TM}}$ Primary Antibody Dilution Buffer for staining (Beyotime Institute of Biotechnology) which were incubated overnight at $4^{\circ} \mathrm{C}$. The sections were then incubated with an Alexa Fluor ${ }^{\circledR} 488$-conjugated anti-rabbit $\operatorname{IgG}(\mathrm{H}+\mathrm{L}), \mathrm{F}\left(\mathrm{ab}^{\prime}\right) 2$ fragment antibody (cat. no. 4412S, 1:1,000, Cell Signaling Technology, Inc.) for $1 \mathrm{~h}$, stained with 4',6-diamidino-2-phenylindole (DAPI) for $6 \mathrm{~min}$, and imaged using a fluorescence microscope. A total of three sections were selected for each time point (24, 48 and $72 \mathrm{~h})$ in each group and three visual fields were randomly selected for each section. ImageJ software was used to measure IOD values for each condition.

Ultrastructure assessment of lung injury. Following fixation, the isolated lung tissues were rinsed in $0.1 \mathrm{~mol} / 1$ phosphate buffer ( $\mathrm{pH}$ 7.4) and dehydrated in a graded ethanol series. The tissues were subsequently infiltrated and embedded in epoxy-resin at $60^{\circ} \mathrm{C}$ for $48 \mathrm{~h}$. The ultrathin tissue sections $(70 \mathrm{~nm})$ were stained using uranyl acetate and lead citrate at $20^{\circ} \mathrm{C}$ and then examined using transmission electron microscopy (Hitachi-HT7700; Hitachi, Ltd.).

Statistical analysis. Statistical analysis was performed using SAS 9.4 software (SAS Institute, Inc.). The statistical description of quantitative variables was expressed as mean \pm standard deviation. Analysis between different treatment groups and time points was performed using factorial design ANOVA followed by the LSD-t test post hoc test. $\mathrm{P}<0.05$ was considered to indicate a statistically significant difference.

\section{Results}

Establishment of the immunosuppressed guinea pig model. Consistent with the results of Kirkpatrick et al (11), in the present study, treatment with a combination of triamcinolone acetonide and cyclophosphamide was used to establish a transient immunosuppressed guinea pig model. The white blood cell, neutrophil and lymphocyte counts of immunosuppressed guinea pigs were significantly reduced compared with Control (Table I).

Lung tissue injury is more severe in the immunosuppressed Lp-infected group compared with the Lp-infected group with increasing infection time. At each time point, the lung tissue structure of the Control group was normal (Fig. 2A). As the infection time increased from 24 to $72 \mathrm{~h}$, the H\&E-stained lung tissue samples from the Lp-infected group exhibited progressive damage, with the most prominent observed at $72 \mathrm{~h}$. After $72 \mathrm{~h}$ infection, the tissue structure was disordered with the structures of the alveoli and alveolar walls becoming particularly unclear and a large number of inflammatory cell infiltration (mainly neutrophils; Fig. 2C and E). In tissues from the immunosuppressed Lp-infected group at the same timepoint, the pathological changes in the lung tissues were more aggravated with extensive structural disorder. A large number of alveoli were filled with inflammatory exudate, the alveolar walls were extensively thickened and reductions in the number of alveoli was observed (Fig. 2D and F). At 72 h, the infection area of $\mathrm{Lp}$ infected group was larger compared with that of the immunosuppressed Lp-infected group, but the damage was not as extensive (Fig. 2G). A small level neutrophil infiltration was observed in the alveolar walls of the immunosuppressed group at $24 \mathrm{~h}$ after infection (data not shown), and the lung tissues then largely returned to normal at $72 \mathrm{~h}$ (Fig. 2B).

As the infection time increased, the ultrastructure of the lung tissue from the Control group was normal (Fig. 3A), whereas that from the Lp-infected group was aggravated, 

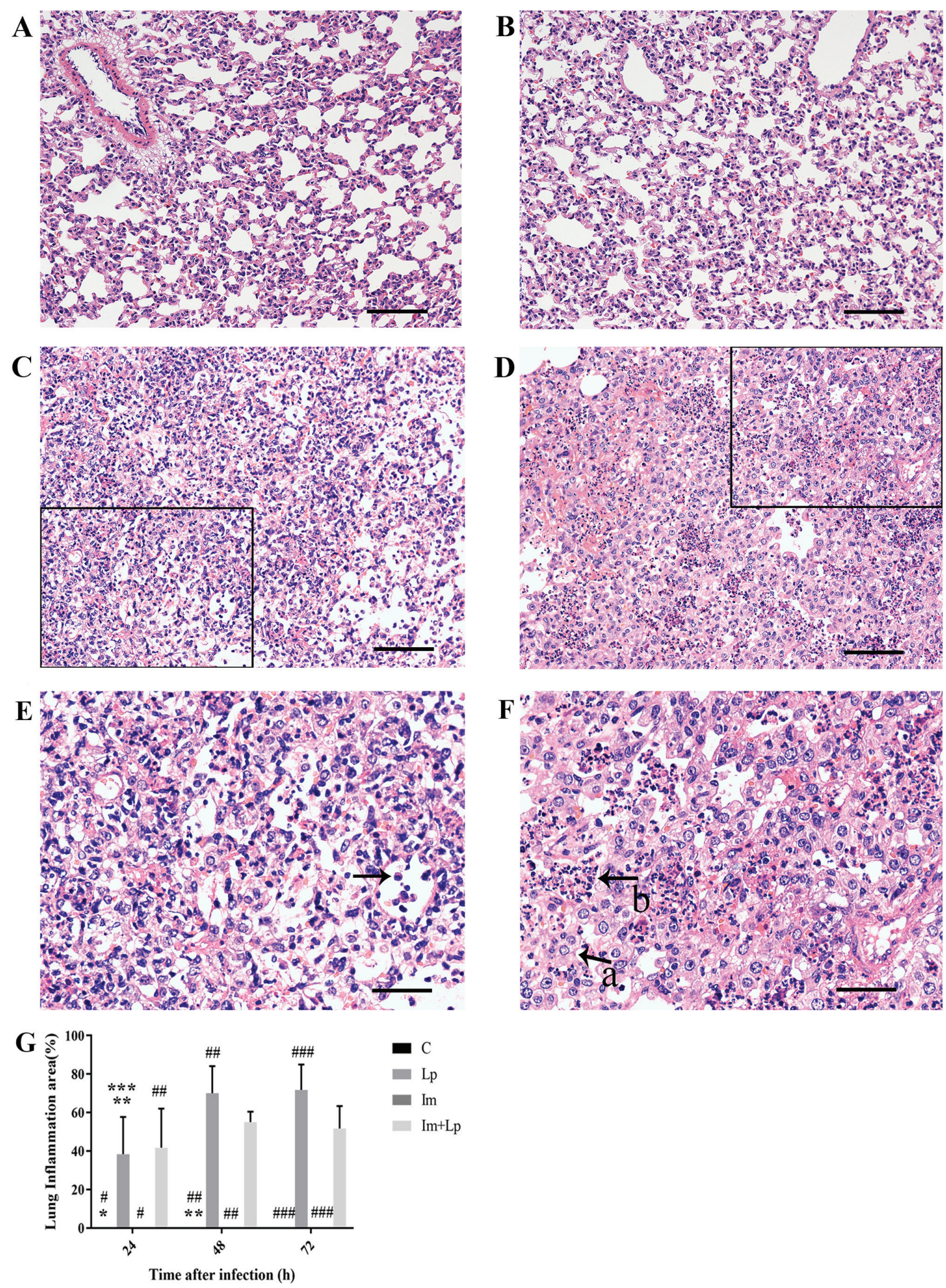

Figure 2. H\&E staining of lung tissue sections $72 \mathrm{~h}$ after Lp infection. (A) In the control group, the tissue structure was normal. (B) In the immunosuppressed group, the tissue structure was normal. (C and E) In the Lp-infected group, the tissue structure was more disordered with the alveoli and alveolar walls unclear and a large number of inflammatory cells were visible (indicated by arrow). (E) is enlargement of the black box of (C). (D and F) In the immunosuppressed Lp-infected group, the alveolar walls were extensively thickened with the total number of alveoli was reduced (indicated by arrow a), extensive structural disorder was observed and a large number of alveoli were filled with inflammatory exudate (indicated by arrow b). (F) is enlargement of the black box of (D). (G) Quantified lung inflammation area (\%). Scale bar, $100 \mu \mathrm{m}$ (A-D) and $50 \mu \mathrm{m}$ (E and F) ${ }^{*} \mathrm{P}<0.05$ vs. Lp $24 \mathrm{~h} ;{ }^{* *} \mathrm{P}<0.05$ vs. Lp $48 \mathrm{~h} ;{ }^{* * * *} \mathrm{P}<0.05$ vs. Lp $72 \mathrm{~h}$; ${ }^{\#} \mathrm{P}<0.05$ vs. Im + Lp 24 h; ${ }^{\# \#} \mathrm{P}<0.05$ vs. Im + Lp 48 h; and ${ }^{\# \# \# ~} \mathrm{P}<0.05$, vs. Im + Lp 72 h. Lp, Legionella pneumophila; Im, immunosuppressed.

with the structure of the lung tissue becoming disordered observed at $72 \mathrm{~h}$ (Fig. 3B). The surfaces of the alveolar capillary endothelial cells and alveolar epithelial cells were uneven and the basement membranes between them becoming 


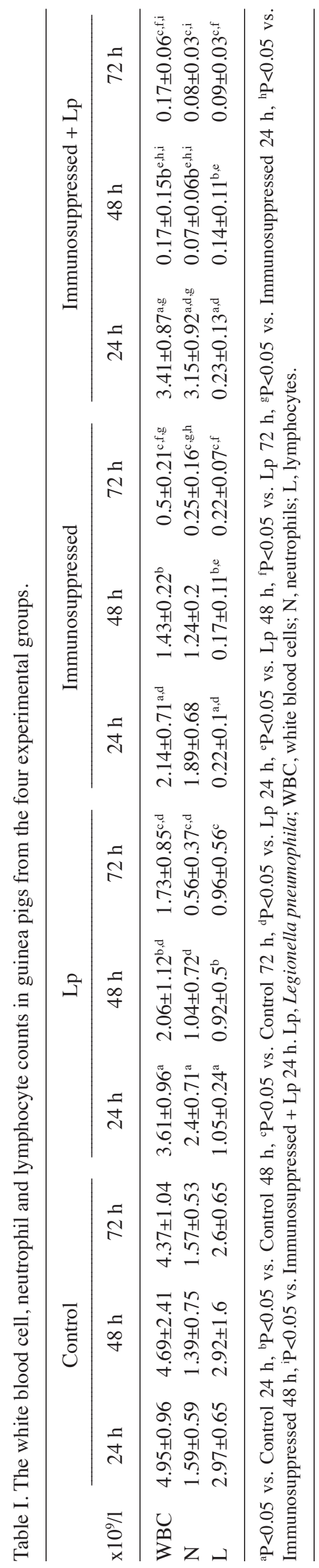
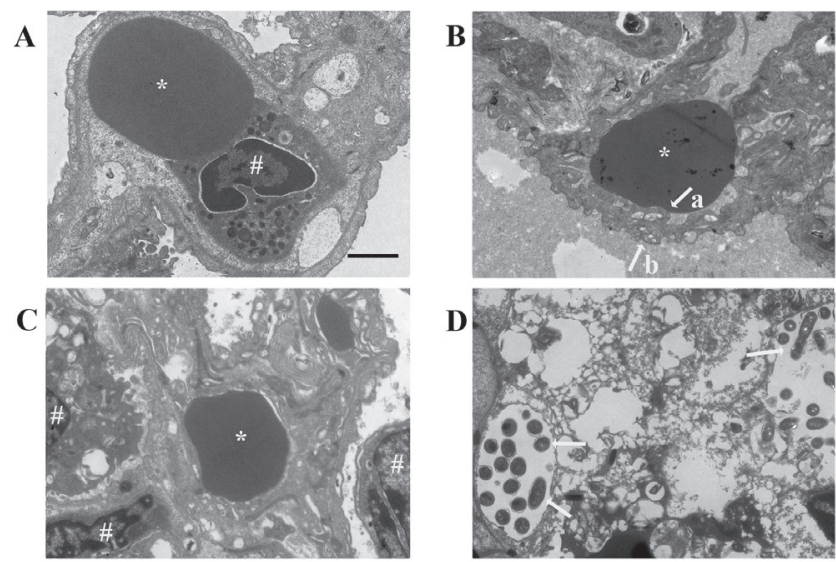

Figure 3. Electron micrographs of Lp-infected guinea pig lung tissues $72 \mathrm{~h}$ after infection. (A) Ultrastructure in the lung tissues from the control group was normal. (B) In the Lp-infected group, the lung tissue was disordered. The surfaces of pulmonary capillary endothelial cells (indicated by arrow a) and alveolar epithelial cells (indicated by arrow b) were uneven, and the basement membrane between the two was blurred. (C) In the immunosuppressed group, the ultrastructure was close to normal. (D) In the lung tissues from the immunosuppressed Lp-infected group, destruction to the tissue structure was extensive and severe, with larger quantities of Lp infiltration (indicated by arrows). * denotes red blood cell and \# denotes nucleus. Scale-bar, $2 \mu \mathrm{m}$. Lp, Legionella pneumophila.

blurred. This damage was more severe in the lung tissues from the immunosuppressed Lp-infected group, which exhibited extensive damage to the lung tissue structure at $72 \mathrm{~h}$ with a certain amount of Lp bacterial infiltration (Fig. 3D). In the immunosuppressed group, the capillary endothelial cells were swollen at $24 \mathrm{~h}$ and the mitochondria had degenerated. As the infection time increased, the ultrastructure of the lung tissue gradually recovered to normal (Fig. 3C).

The weight of lungs in animals from the immunosuppressed Lp-infected group increases gradually with infection time, but is less compared with that in the Lp-infected group at $72 \mathrm{~h}$. The severity of pulmonary edema was assessed by measuring the weights of lungs from the animals at different time points. At $72 \mathrm{~h}$, the mean weight of the lungs was greater in the Lp-infected group compared with that in the immunosuppressed Lp-infected group $(\mathrm{P}<0.05)$, whereas that in the immunosuppressed group was only slightly higher compared with that in the control group, but there was no significant difference (Fig. 4A).

The weights of the lungs from the immunosuppressed Lp-infected group first decreases and then increases as the infection time increased. As the infection time increased, under perfusion at constant pressure, the weights of the ex vivo lungs from the Lp-infected group increased, with this gain being most significant at $48 \mathrm{~h}$ (Fig. 4E). In the immunosuppressed Lp-infected group, the weights of the ex vivo lungs first decreased following $24 \mathrm{~h}$ but then gradually increased from $48 \mathrm{~h}$ onwards and finally the increase at $72 \mathrm{~h}$ was greater compared with the immunocompetent Lp-infected group $(\mathrm{P}<0.05 ;$ Fig. 4B-E). The weights of the ex vivo lungs in the immunosuppressed group increased at all time points, but the rate of increase gradually decreased (Fig. 4B-E). The changes in the weights of the ex vivo 
A

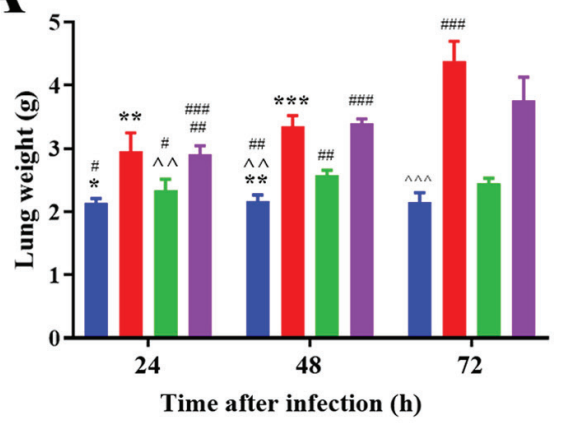

C

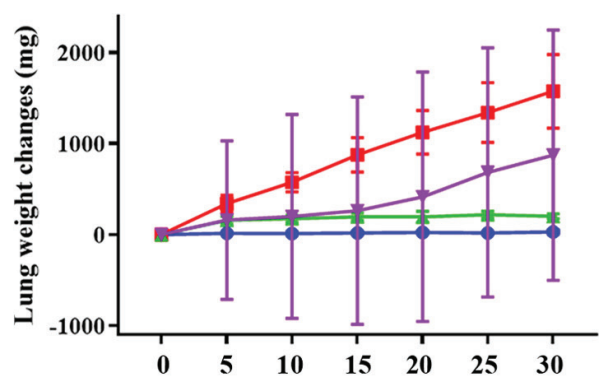

Constant-pressure perfusion time (min)
B
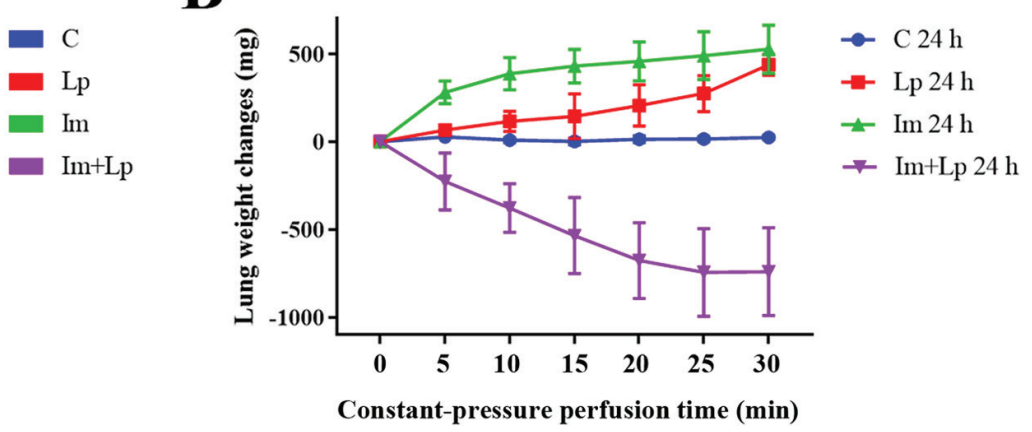

D

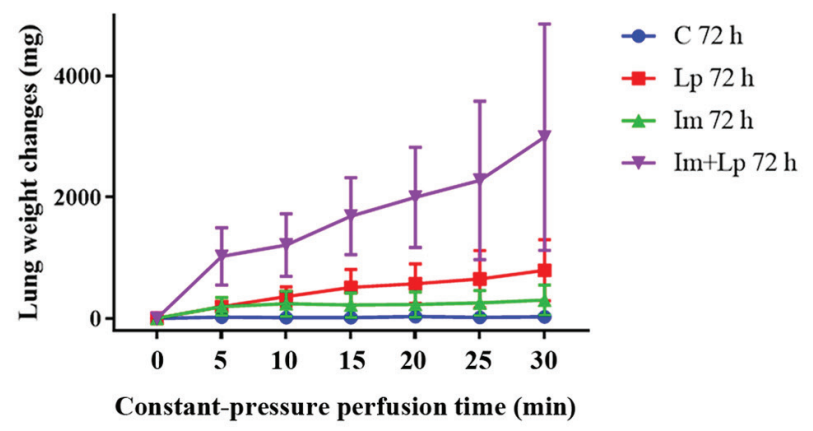

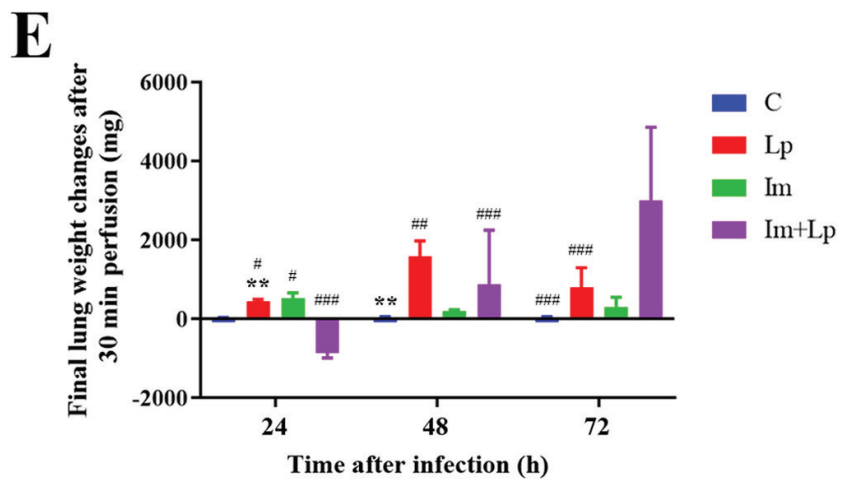

Figure 4. Actual lung weight in vivo and changes of lung weights in vitro following Lp infection. (A) Weights of lungs from each experimental group at 24 , 48 and $72 \mathrm{~h}$ following Lp infection. (B) Changes in the weights of lungs from each experimental group at $24 \mathrm{~h}$ during $30 \mathrm{~min}$ of isolated lung perfusion. (C) Changes in the weights of lungs from each experimental group at $48 \mathrm{~h}$ during $30 \mathrm{~min}$ of isolated lung perfusion. (D) Changes in the weights of lungs from each experimental group at $72 \mathrm{~h}$ during $30 \mathrm{~min}$ of isolated lung perfusion. (E) Final changes in the weights of lungs from each experimental group at 24,48 and $72 \mathrm{~h}$ after $30 \mathrm{~min}$ of isolated lung perfusion. ${ }^{*} \mathrm{P}<0.05$ vs. Lp $24 \mathrm{~h} ;{ }^{* *} \mathrm{P}<0.05$ vs. Lp $48 \mathrm{~h} ;{ }^{* * *} \mathrm{P}<0.05$ vs. Lp $72 \mathrm{~h} ;{ }^{\wedge} \mathrm{P}<0.05$ vs. Im $48 \mathrm{~h} ;{ }^{\wedge \wedge} \mathrm{P}<0.05 \mathrm{vs}$. Im $72 \mathrm{~h}$; ${ }^{\#} \mathrm{P}<0.05$ vs. Im + Lp 24 h; ${ }^{\# \# P<0.05 ~ v s . ~ I m ~+~ L p ~} 48$ h; and ${ }^{\# \# "} \mathrm{P}<0.05$ vs. Im + Lp 72 h. C, control; Lp, Legionella pneumophila; Im, immunosuppressed.

lungs were significantly greater in Lp-infected guinea pigs compared with uninfected guinea pigs $(\mathrm{P}=0.0008)$, and the change exhibited by immunosuppressed guinea pigs was significantly greater compared with those without immunosuppression $(\mathrm{P}<0.05)$. Therefore, this observation indicates a synergistic effect between infectiousness and immunosuppression $(\mathrm{P}<0.05)$.

Ultrastructural damage to endothelial junctions of pulmonary capillaries is significantly greater in the immunosuppressed Lp-infected group. The lung capillary endothelial cells were swollen in the lung tissues from the Lp-infected group $24 \mathrm{~h}$ following infection with the cell junctions partially open (Fig. 5); at 48 h, the capillary endothelial cell junctions were completely open before finally at $72 \mathrm{~h}$, the junctions were blurred and partially open (Fig. 5). In the lung tissues from the immunosuppressed Lp-infected group, the capillary endothelial cells were swollen with a reduction in the density and number of cell junctions $24 \mathrm{~h}$ after infection, which were already partially open (Fig. 5); at $48 \mathrm{~h}$, the number of capillary endothelial cell junctions had decreased further with their densities was intermittently reduced, which were partially open with scattered Lp bacterium visible (Fig. 5). Finally, at $72 \mathrm{~h}$ the lung tissue was notably damaged with the cell membranes of the capillary endothelial cells no longer resolvable, the nuclei were swollen and the cell junctions disappeared (Fig. 5). In the lung tissues from the immunosuppressed group, the capillary endothelial cell junctions were partially open at $24 \mathrm{~h}$ (Fig. 5); at $48 \mathrm{~h}$ the density of cell junctions had decreased and were intermittently opened (Fig. 5). However, the endothelial cell junctions had recovered approach to normal by $72 \mathrm{~h}$ (Fig. 5). 
$24 \mathrm{~h}$
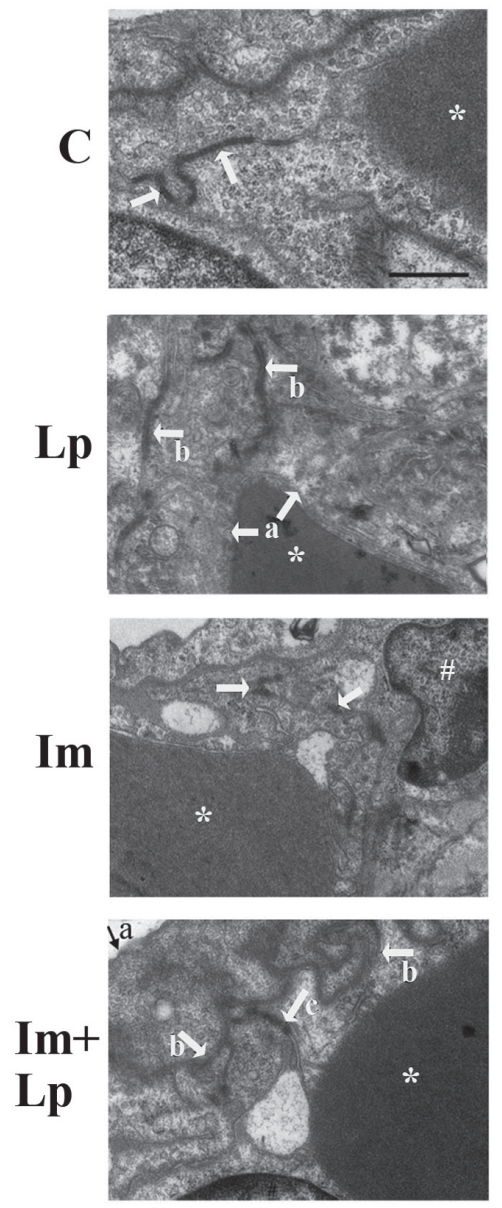

$48 \mathrm{~h}$
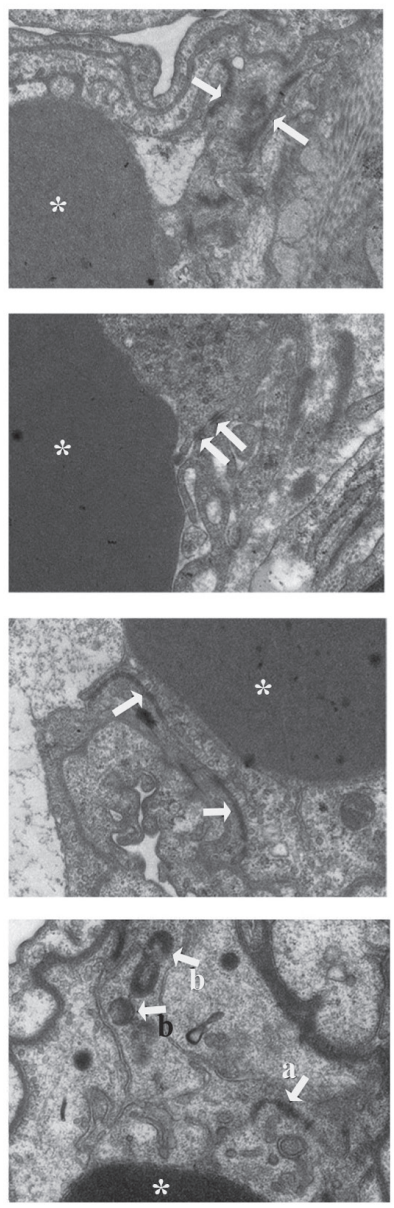

$72 \mathrm{~h}$
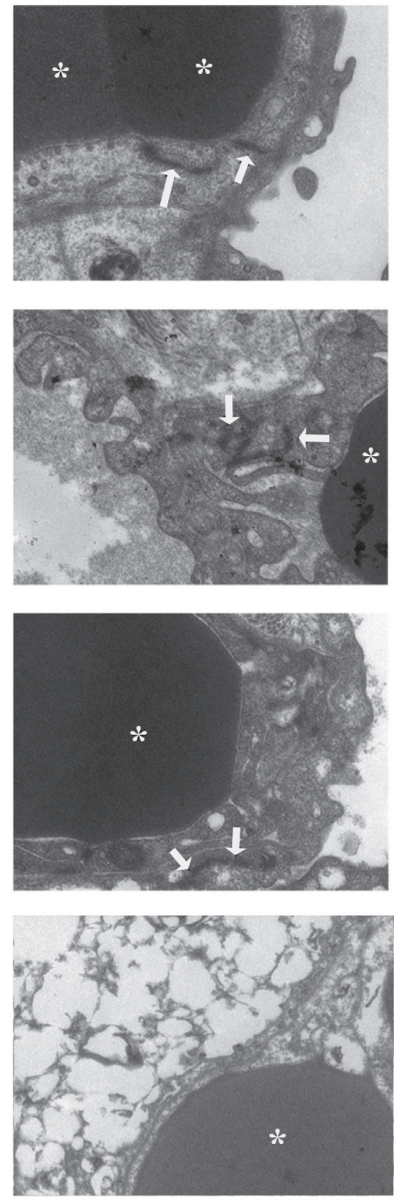

Figure 5. Transmission electron microscopy images of pulmonary capillary endothelial cell junctions in lung tissues isolated from guinea pigs from the experimental groups. Pulmonary capillary endothelial cell junctions in the control group were normal (indicated by arrows). In the Lp-infected group, at 24 h, abnormal changes that could be observed included swollen vascular endothelial cells (indicated by arrows a) and partially opened cell junctions (indicated by arrows b); at $48 \mathrm{~h}$, the endothelial cell junctions were completely opened (indicated by arrows); at $72 \mathrm{~h}$, the cell junctions were obscure and partially opened (indicated by arrows). In the immunosuppressed group, at $24 \mathrm{~h}$, the endothelial cell junctions were partially opened (indicated by arrows); at $48 \mathrm{~h}$, the density of the cell junctions was reduced and the cell junctions were intermittently opened (indicated by arrows); at $72 \mathrm{~h}$, the endothelial cell junctions had recovered to normal (indicated by arrows). In the immunosuppressed Lp-infected group, at $24 \mathrm{~h}$, the alveolar epithelial cells were swollen (indicated by arrow a), the basement membrane was shrunken (indicated by arrows b), the density and number of cell junctions were reduced and the junctions were partially opened (indicated by arrow c); at $48 \mathrm{~h}$, the number and densities of cell junctions were reduced and partially opened (indicated by arrow a), Lp bacteria were visible (indicated by arrows b); at $72 \mathrm{~h}$, destruction of tissue structure was evident, the cell membrane was unclear, the nuclei were swollen, and the cell junctions had disappeared. "Denotes red blood cells and "denotes the nucleus. Scale-bars, $1 \mu \mathrm{m}$. C, control; Lp, Legionella pneumophila; Im, immunosuppressed.

VE-cadherin expression is significantlylower in the lung tissues from the immunosuppressed Lp-infected group compared with the Lp-infected group. An immunofluorescence assay of VE-cadherin in the lung tissues showed that the integral optical density (IOD) value for VE-cadherin fluorescence in the lung tissues from the immunosuppressed Lp-infected group was gradually decreased from 24 to $72 \mathrm{~h}$ and was lower compared with the Lp-infected group at each time point $(\mathrm{P}<0.05$; Fig. $6 \mathrm{~A}$ and $\mathrm{B}$ ). The expression of VE-cadherin in lung tissues was also measured using western blot analysis (Fig. S3). The expression of VE-cadherin appeared to be reduced in Lp-infected group and immunosuppressed Lp-infected group, but no significant differences between each group were observed.

\section{Discussion}

Lp infection is more likely to result in severe pneumonia in immunosuppressed hosts compared with immunocompetent hosts $(2,4)$. The typical pathological changes in severe pneumonia are increased PCP and the consequent formation of pulmonary edema. In the present study, using H\&E staining and transmission electron microscopy, it was found that the opening of capillary endothelial cell junctions and the injury to the lung tissue were more serious in immunosuppressed guinea pigs infected with Lp in compared with in the animals infected with Lp alone, despite the area of lung inflammation being larger in the latter. This is consistent with a previous clinical report that $\mathrm{Lp}$ infection is positively associated with the severity of the condition in immunosuppressed hosts compared within normal hosts (9).

Existing methods used to study PCP, including the measurement of Evans blue content, fluorescein-isothiocyanate (FITC)-labeled BSA and the ratio of albumin in the plasma to that in the alveolar lavage fluid, are indirect detection methods that are unsuitable for severe cases due to the fact they are susceptible to interference by other factors such as 

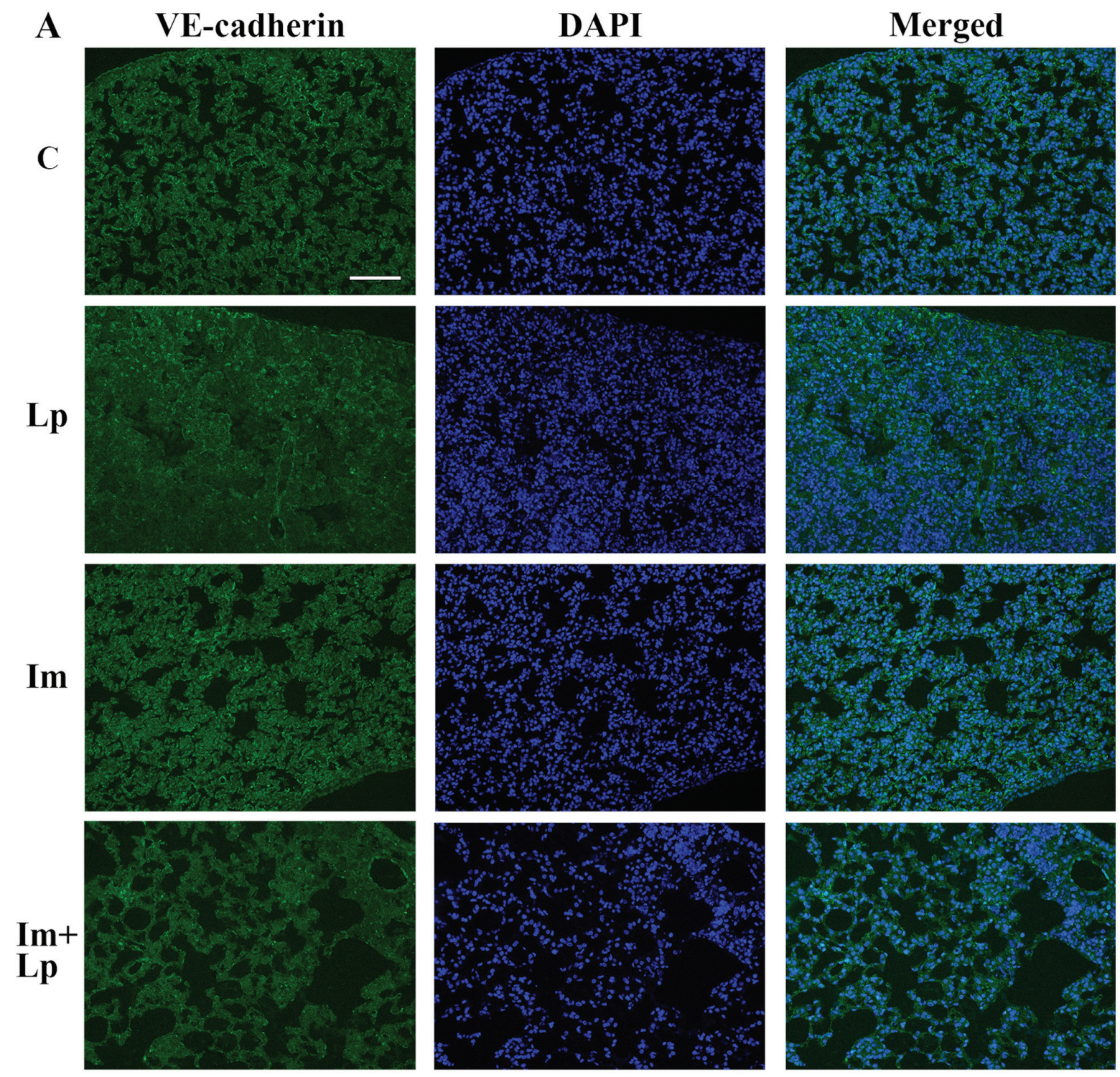

\section{B}

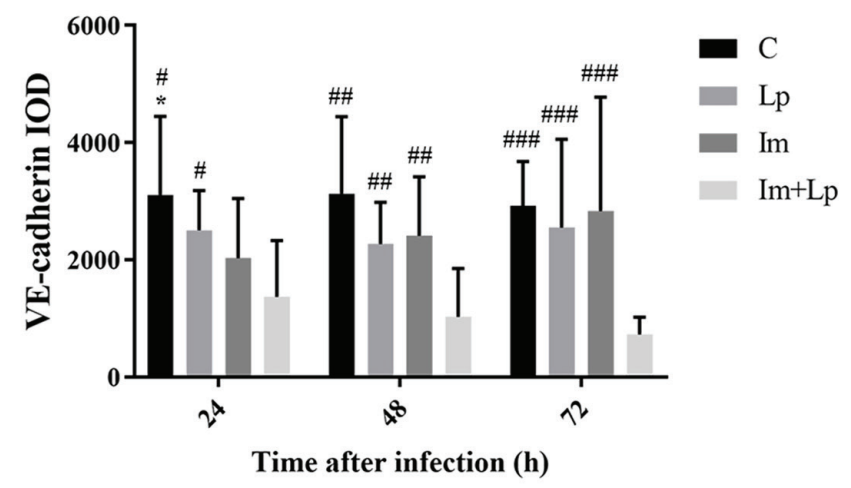

Figure 6. Expression of VE-cadherin detected in lung tissues isolated from guinea pigs from the experimental groups using immunofluorescence staining. (A) Representative images of VE-cadherin staining in lung tissues isolated from guinea pigs from the four experimental groups $72 \mathrm{~h}$ after infection. (B) IOD of VE-cadherin fluorescence as calculated from the images demonstrates that VE-cadherin expression in the immunosuppressed Lp-infected group was the lowest compared with all other groups. ${ }^{*} \mathrm{P}<0.05$ vs. Im 24 h; ${ }^{\#} \mathrm{P}<0.05$ vs. Im + Lp 24 h; ${ }^{\# \#} \mathrm{P}<0.05$ vs. Im + Lp 48 h; and ${ }^{\# \# / ~ P<0.05 ~ v s . ~ I m ~+~ L p ~} 72$ h. Scale-bar, $100 \mu \mathrm{m}$. C, control; Lp, Legionella pneumophila; Im, immunosuppressed; VE-cadherin, vascular-endothelial cadherin; IOD, integral optical density.

hypoalbuminemia (18). Wang et al (18) used an Evans blue assay to compare the changes in PCP in Streptococcus pneumonia-infected mice with normal immunity and mice with cyclophosphamide-induced leukopenia at different time points $(4,24,48,72$ and $96 \mathrm{~h})$. They found that the leukopenic group exhibited higher PCP compared with the normal immunity group only at $48 \mathrm{~h}$. The present study focused on the application of IPL-2 Isolated Perfused Lung System to remove the influence of extrapulmonary factors to directly measure PCP in guinea pigs infected with $\mathrm{Lp}$. 
In the present study, the IPL-2 Isolated Perfused Lung System was used to study the effects of immunosuppression and infection on PCP over time. This system has two modes of perfusion, constant-pressure and constant-current perfusion. The former not only simulates the in vivo lung circulation, but also excludes the influence of extrapulmonary factors $(13,14)$. The changes in the weights of lungs ex vivo directly reflects changes in PCP, which can be used to evaluate PCP in conditions in which the degree of lung injury varies $(13,14)$. In the control group, PCP was normal and the weights of the lungs ex vivo exhibited no notable changes under constant-pressure perfusion mode.

However, as the infection time increased, the weights of the lungs ex vivo in the immunosuppressed Lp-infected group did not continue to increase; instead, the weight of the lungs gradually changed from displaying a significant reduction to a significant increase. These changes could be caused by a gradual increase in PCP in this group. Another important cause could be that the average PAP in the Lp-infected immunosuppressed guinea pigs gradually changed from significantly higher to lower compared with the normal average pressure. At $24 \mathrm{~h}$ after infection, PCP increased in the Lp-infected immunosuppressed guinea pigs, by which time the average PAP was already significantly higher compared with the normal pressure in vivo. When the lung was perfused with normal average PAP, the pulmonary capillary hydrostatic pressure decreased relative to the in vivo state, causing the edema fluid flowed out of the lung into the perfusate, reducing the weights of the lungs ex vivo. PCP increased further $48 \mathrm{~h}$ after infection, whereas in vivo, the average PAP was lower compared with $24 \mathrm{~h}$. It was considered that at $48 \mathrm{~h}$, the average PAP in the Im+Lp group in vivo remained higher compared with the controls in some animals, but in other animals, the average PAP had decreased to below the normal level. Therefore, under perfusion with normal average PAP, the ex vivo weight of the lungs both increased and decreased in the Lp-infected immunosuppressed animals at $48 \mathrm{~h}$. At $72 \mathrm{~h}, \mathrm{PCP}$ was significantly increased, whilst in vivo, the average PAP was below the normal level and the pulmonary capillary hydrostatic pressure had decreased. This led to insufficient lung perfusion and a lower weight of the lungs in the immunosuppressed Lp-infected group compared with in the Lp-infected group. Perfusion with the normal average PAP elevated the pulmonary capillary hydrostatic pressure and significantly increased the ex vivo weight of the lungs. It has been clinically observed that immunosuppressive therapy can reduce the average PAP in patients with pulmonary hypertension caused by systemic lupus erythematosus or mixed connective tissue disease $(19,20)$, suggesting that immunosuppressive therapy may also reduce the average PAP in hosts with normal immunity. However, the reason for the mean PAP being elevated in the early stages of infection in the Lp-infected immunosuppressed group requires further study.

The vascular endothelial cell junction protein VE-cadherin serves an important role in the regulation of the vascular endothelial cell barrier function, where its internalization increases PCP (21-24). In the presence of inflammation, activated neutrophils accumulate in the microvasculature and release proteolytic enzymes (25). This enzyme cleaves the extracellular region of VE-cadherin (26), increasing vascular permeability in the area of leukocyte accumulation. In the present study, immunofluorescence assay showed that the expression of VE-cadherin was lower in the immunosuppressed Lp-infected group compared with that in the Lp-infected alone group. Neutrophil infiltration was observed in both groups from H\&E staining. Electron microscopy showed that as the infection time increased, the damage to the pulmonary capillary endothelial cells was more extensive in the immunosuppressed Lp-infected group compared with in the Lp-infected alone group. Therefore, it could be concluded from these findings that the increase in PAP in the Lp-infected immunosuppressed guinea pigs resulted from the synergistic effects of multiple factors, including the internalization and cleavage of VE-cadherin and the tissue damage caused by Lp. Pulmonary inflammation was heterogeneous throughout the tissue. Therefore, the immunofluorescence staining of VE-cadherin in the lung tissues from the Lp-infected and immunosuppressed Lp-infected groups was non-uniform, which could provide an explanation for the large variation observed in the corresponding VE-cadherin western blots of the lung tissues (Fig. S3). It was hypothesized in the present study that the distribution of VE-cadherin could be observed more accurately in the tissue using immunofluorescence compared with Western blot, where errors could be reduced. Therefore, immunofluorescence was used to detect the expression of VE-cadherin. The mechanism underlying the reduction of VE-cadherin expression remains unclear and require further study, along with changes in pulmonary vascular resistance and the pulmonary artery pressure in response to Lp infection.

Pentobarbital is not suitable for use as an anesthetic in guinea pigs as it can cause cardiovascular and respiratory depression, however, these side effects can be avoided by using the IPL-2 Isolated Perfused Lung System $(27,28)$. This is because the ventilation mode of this system is by tracheal intubation and the application of negative pressure ventilation $\left(-2-10 \mathrm{cmH}_{2} \mathrm{O}\right)$, with the respiration of this isolated lung carried out only under this negative pressure. The heart only acts as a vehicle for intubation and therefore does not participate in pulmonary circulation under this system.

The application of IPL-2 Isolated Perfused Lung System requires substantial preparation (procedures including preparation of perfusion fluid, cardiopulmonary extraction and machine operation), which can potentially lead to a loss of time required for the detection of bacterial load. There have been numerous reports of Legionella pneumophila infection in laboratory animals, where the correlation between the bacterial concentration in the lungs and the severity of pneumonia is low $(12,29,30)$. Therefore, the bacterial load in the lungs in the present study was not measured.

In the immunosuppressed Lp-infected group, under constant-pressure perfusion, the weight of the lungs ex vivo decreased significantly $24 \mathrm{~h}$ after infection, but increased significantly after $72 \mathrm{~h}$, indicating that PCP increased in the immunosuppressed Lp-infected hosts, and that the average PAP also had a profound effect on the degree of 
in vivo pulmonary edema. Indeed, Lp infection in immunosuppressed hosts is more likely to develop into severe pneumonia. Most patients with severe pneumonia receive intensive therapies, including mechanical ventilation and vasoactive drugs, leading to an elevation in PAP and the aggravation of pulmonary edema $(31,32)$. Therefore, data from the present study suggest that during the intensive treatment of Lp infection immunosuppressed patients, PCP will increase and therefore the PAP should be monitored for each patient to avoid aggravating pulmonary edema.

\section{Acknowledgements}

Not applicable.

\section{Funding}

The present study was supported by the National Key R\&D Program of China (grant no. 2017YFC1309702) and the National Natural Science Foundation of China (grant no. 81170009).

\section{Availability of data and materials}

All data generated or analyzed in this study are included in this published article.

\section{Authors' contributions}

JK, YC, WW and XC designed the experiments, and XC, NY, JM, WYL and MX performed them. EL and MZ collected and analyzed the data. XC wrote the manuscript. All authors read and approved the final manuscript.

\section{Ethics approval and consent to participate}

The Experimental Animal Welfare and Ethics Committee of the Chinese Medical University (Shenyang, China) approved the experimental protocols.

\section{Patient consent for publication}

Not applicable.

\section{Competing interests}

The authors declare that they have no competing interests.

\section{References}

1. Stout JE and Yu VL: Legionellosis. New Eng J Med 337: 682-687, 1997.

2. Helms CM, Viner JP, Weisenburger DD, Chiu LC, Renner ED and Johnson W: Sporadic legionnaires' disease: Clinical observations on 87 nosocomial and community-acquired cases. Am J Med Sci 288: 2-12, 1984

3. Pedro-Botet ML, Sabria-Leal M, Sopena N, Manterola JM, Morera J, Blavia R, Padilla E, Matas L and Gimeno JM: Role of immunosuppression in the evolution of Legionnaires' disease. Clin Infect Dis 26: 14-19, 1998.

4. Beaute J, Zucs P and de Jong B; European Legionnaires Disease Surveillance Network: Legionnaires disease in Europe, 2009-2010. Euro Surveill 18: 20417, 2013.
5. Clark SB and Soos MP: Noncardiogenic Pulmonary Edema. In: StatPearls. StatPearls Publishing LLC., Treasure Island (FL), 2019. https://www.ncbi.nlm.nih.gov/books/NBK542230/

6. Htwe TH and Khardori NM: Legionnaire's disease and immunosuppressive drugs. Infect Dis Clin North Am 31: 29-42, 2017.

7. Lo SK, Everitt J, Gu J and Malik AB: Tumor necrosis factor mediates experimental pulmonary edema by ICAM-1 and CD18-dependent mechanisms. J Clin Invest 89: 981-988, 1992.

8. Donners MM, Wolfs IM, Olieslagers S, Mohammadi-Motahhari Z, Tchaikovski V, Heeneman S, van Buul JD, Caolo V, Molin DG, Post MJ and Waltenberger J: A disintegrin and metalloprotease 10 is a novel mediator of vascular endothelial growth factor-induced endothelial cell function in angiogenesis and is associated with atherosclerosis. Arterioscler Thromb Vasc Biol 30: 2188-2195, 2010.

9. Edelstein PH: Legionella jamestowniensis fatal pneumonia in an immunosuppressed man. J Infect Chemother 23: 59-61, 2017.

10. Nanovic Z: Legionnaires' disease and use of tumor necrosis factor- $\alpha$ lpha inhibitors: A forthcoming problem? Macedonian. J Med Sci 6: 465-472, 2013

11. Kirkpatrick WR, McAtee RK, Fothergill AW, Rinaldi MG and Patterson TF: Efficacy of voriconazole in a guinea pig model of disseminated invasive aspergillosis. Antimicrob Agents Chemother 44: 2865-2868, 2000.

12. Gamradt P, Xu Y, Gratz N, Duncan K, Kobzik L, Högler S, Kovarik P, Decker T and Jamieson AM: The influence of programmed cell death in myeloid cells on host resilience to infection with legionella pneumophila or streptococcus pyogenes. PLoS Pathog 12: e1006032, 2016.

13. Ismael-Badarneh R, Guetta J, Klorin G, Berger G, Abu-Saleh N, Abassi Z and Azzam ZS: The role of angiotensin II and cyclic AMP in alveolar active sodium transport. PLoS One 10: e0134175, 2015.

14. Ong HX, Benaouda F, Traini D, Cipolla D, Gonda I, Bebawy M, Forbes B and Young PM: In vitro and ex vivo methods predict the enhanced lung residence time of liposomal ciprofloxacin formulations for nebulisation. Eur J Phar Biopharm 86: 83-89, 2014.

15. Kadlecek S, Shaghaghi H, Siddiqui S, Profka H, Pourfathi M and Rizi R: The effect of exogenous substrate concentrations on true and apparent metabolism of hyperpolarized pyruvate in the isolated perfused lung. NMR Biomed 27: 1557-1570, 2014.

16. Dominguez-Fandos D, Valdes C, Ferrer E, Puig-Pey R, Blanco I, Tura-Ceide O, Paul T, Peinado VI and Barberà JA: Sildenafil in a cigarette smoke-induced model of COPD in the guinea-pig. Eur Respir J 46: 346-354, 2015.

17. He X, Liang Y, LaValley MP, Lai J and Ingalls RR: Comparative analysis of the growth and biological activity of a respiratory and atheroma isolate of chlamydia pneumoniae reveals strain-dependent differences in inflammatory activity and innate immune evasion. BMC Microbiol 15: 228, 2015.

18. Wang E, Simard M, Ouellet N, Bergeron Y, Beauchamp D and Bergeron MG: Pathogenesis of pneumococcal pneumonia in cyclophosphamide-induced leukopenia in mice. Infect Immun 70: 4226-4238, 2002

19. Machireddy K, Myint Z, Dein E, Mathai SC, Seo P, Haque U, Manno R and Timlin H: Mycophenolate mofetil in a lupus patient with pulmonary hypertension. Cureus 10: e2121, 2018.

20. Jais X, Launay D, Yaici A, Le Pavec J, Tchérakian C, Sitbon O, Simonneau $\mathrm{G}$ and Humbert M: Immunosuppressive therapy in lupus- and mixed connective tissue disease-associated pulmonary arterial hypertension: A retrospective analysis of twenty-three cases. Arthritis Rheum 58: 521-531, 2008.

21. Bates DO: Vascular endothelial growth factors and vascular permeability. Cardiovas Res 87: 262-271, 2010.

22. Dejana E and Giampietro C: Vascular endothelial-cadherin and vascular stability. Curr Opin Hematol 19: 218-223, 2012.

23. Komarova Y and Malik AB: Regulation of endothelial permeability via paracellular and transcellular transport pathways. Ann Rev Physiol 72: 463-493, 2010.

24. Komarova YA, Mehta D and Malik AB: Dual regulation of endothelial junctional permeability. Sci STKE 2007: re8, 2007.

25. Weiss SJ: Tissue destruction by neutrophils. N Engl J Med 320: 365-376, 1989 .

26. Lampugnani MG, Resnati M, Raiteri M, Pigott R, Pisacane A, Houen G, Ruco LP and Dejana E: A novel endothelial-specific membrane protein is a marker of cell-cell contacts. J Cell Biol 118: 1511-1522, 1992.

27. Lan CC, Peng CK, Tang SE, Wu SY, Huang KL and Wu CP: Anti-vascular endothelial growth factor antibody suppresses ERK and NF- $\mathrm{KB}$ activation in ischemia-reperfusion lung injury. PLoS One 11: e0159922, 2016. 
28. Rieg AD, Suleiman S, Perez-Bouza A, Braunschweig T, Spillner JW, Schröder T, Verjans E, Schälte G, Rossaint R, Uhlig S and Martin C: Milrinone relaxes pulmonary veins in guinea pigs and humans. PLoS One 9: e87685, 2014.

29. Viswanathan VK, Edelstein PH, Pope CD and Cianciotto NP: The legionella pneumophila iraAB locus is required for iron assimilation, intracellular infection, and virulence. Infect Immun 68: 1069-1079, 2000.

30. Edelstein PH: The guinea pig model of legionnaires' disease. Methods Mol Biol 954: 521-540, 2013.

31. Wang X, Ma S, Liu Y, Xu W and Li Z: Effects and mechanism analysis of combined infusion by levosimendan and vasopressin on acute lung injury in rats septic shock. Cell Biochem Biophys 70: 1639-1645, 2014
32. Daudel F, Tuller D, Krahenbuhl S, Jakob SM and Takala J: Pulse pressure variation and volume responsiveness during acutely increased pulmonary artery pressure: An experimental study. Crit Care 14: R122, 2010

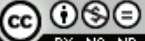

This work is licensed under a Creative Commons Attribution-NonCommercial-NoDerivatives 4.0 International (CC BY-NC-ND 4.0) License. 